\title{
Wird das Abstimmungsresultat unterlaufen?
}

\section{Daniel Bracher}

Präsident «Verein für freie Arztwahl»
Korrespondenz:

Dr. med. D. Bracher Walchstrasse 19

CH-3073 Gümligen

dbracher[at]datacomm.ch
Aus verschiedenen Regionen wurde uns gemeldet, die Krankenkassen würden trotz der Abstimmungsniederlage Netze auf unzulässige Weise fördern: Dort, wo sie Verträge mit Budgetnetzen geschlossen hätten, würden sie diese den Versicherten unter der Bezeichnung «Hausarztmodelle» unterjubeln und die ursprüngliche und von der Bevölkerung unter Hausarztmodell verstandene Form nicht mehr anbieten. Wir sind der Sache nachgegangen und können berichten:

- Eine solche «gemeinsame» Politik aller Krankenkassen gibt es nicht.

- Es gibt zwei grosse Kassen (Helsana und Swica), die in der ganzen Schweiz nirgends ursprüngliche Hausarztmodelle anbieten, sondern nur in ausgewählten Regionen Netzverträge. Das ist rechtens; denn die Kassen sind frei, ob, und wenn ja, welche Sparmodelle sie anbieten wollen. Wir würden es allerdings begrüssen, wenn im Gegenzug andere Kassen nur Hausarztmodelle und keine Netzverträge anböten; denn dies würde Marktverzerrungen durch Quersubventionierungen erschweren.
- und im Falle einer Überweisung an einen Spezialarzt für die Patientin die freie Arztwahl besteht.

- Dort, wo die Kasse die Wahl des Hausarztes oder - im Falle der Überweisungen - des Spezialisten einschränkt, ist von «Listenmodell» zu sprechen.

- Dort, wo die Kasse Verträge mit Netzwerken abschliesst, sind diese entweder als «ManagedCare-Verträge» oder als «Netzverträge mit Budgetmitverantwortung der Ärzte» zu bezeichnen; denn der verführerisch tieferen Prämie stehen zwei Nachteile gegenüber, nämlich

- dass bei Überweisungen an Spezialisten die freie Arztwahl eingeschränkt ist,

- und dass die Budgetmitverantwortung die behandelnden Ärzte zu verdeckter Rationierung verleiten kann (Beteiligung am Gewinn).

Alle Einwohner der Schweiz sind durch Gesetz gezwungen, eine Krankenversicherung abzuschliessen und haben dadurch ein Anrecht auf richtige Infor-

\section{Wir sind beunruhigt, dass viele Kassen unter der unverfänglichen Bezeichnung «Hausarztmodelle» Verträge mit Budgetmitverantwortung propagieren.}

- Unsere Vereinigung ist aber sehr beunruhigt darüber, dass viele Kassen unter der unverfänglichen Bezeichnung «Hausarztmodelle» Verträge mit Budgetmitverantwortung propagieren. Wir halten dies für einen Verstoss gegen die Informationspflicht und fordern: Der Versicherte muss wissen, welchen Vertrag er abschliesst, d. h.:

- Die Bezeichnung «Hausarztmodell» darf nur in ihrem ursprünglichen, der Bevölkerung vertrautem Sinn verwendet werden, nämlich dort, wo - die Hausärztin eine reine Steuerungs-(gatekeeper)Funktion wahrnimmt mation. Und die Krankenkassen sollten ein Interesse an ihrem Ruf haben und nicht in den Verdacht geraten, den Versicherten Nachteile bewusst zu verschweigen. Wir sind daher zuversichtlich, dass sich eine korrekte Information durchsetzen wird, aber bis es so weit ist, empfehlen wir Ihnen, Ihre Patienten dazu anzuhalten, bei Abschluss von Sparverträgen genau hinzusehen.

Weitere Details zu diesen Fragen finden Sie auf der homepage www.verein-freie-arztwahl.ch 